\title{
Editoríal
}

\section{Low-calorie sweeteners: is their safety a real issue?}

(C) Springer Healthcare - CEC Editore 2012

Can the low-calorie sweeteners (LCS) currently sold in Europe be considered safe? Different answers to this question can be found according to the source searched, i.e., the scientific literature, word-of-mouth, lay press, Internet, etc. Peer-reviewed papers almost uniformly reassure consumers: the use of authorised LCS, in quantities below the acceptable daily intake (ADI), is not associated with significant negative effects. It is noteworthy that checking the ADI is not the consumer's responsibility. If this was the case, one would need to carefully compute all of the different LCS contained in his/her everyday food. Conversely, the ADI is established and monitored by ad hoc regulatory bodies such as the European Food Safety Authority (EFSA), which calculate "average" consumption (taking into account all the foods in which the use of any individual sweetener is approved) and make sure that their total falls well below the ADI.

If we search the Internet, this reassuring outcome changes completely. One example is that of searching for "aspartame" in www.google.it: more than 1.5 million pages feature this word. The webpage that appears on top of the ranking is that of Wikipedia. This page is clearly not updated, since is still says that "The EFSA and its corresponding US agency FDA have not yet published an opinion on the Ramazzini study of
2007, which reported potential carcinogenicity of aspartame". On the contrary, the results of this study were evaluated a long time ago by both regulatory bodies, which came to the conclusion that-mostly due to methodological issues-they are not relevant for human health; these conclusions have been made public. Of the remaining nine websites, seven (i.e., with the exception of one producer and of the EFSA) cast a negative or very negative light on aspartame. Examples include "a highly toxic molecule", "one of the most dangerous substances in commerce" or "involved in the pathogenesis of multiple sclerosis and systemic lupus". Below Wikipedia, another site features the self-explanatory subtitle "aspartamekills".

The reasons behind such attitudes are to be investigated. Indeed, there is a large difference between official science and the media, as also documented by a recent survey carried out by the Centro per la Documentazione sul Farmaco of the University of Milan. There are likely two main reasons that explain this phenomenon: on one hand there is a perception that LCS are "chemical" synthetic molecules and, as such, intrinsically dangerous because only nature and its products are-by definition-safe and "friendly". On the other hand, there is generally scarce faith in medicine, scientists, science and its institutions. 
The first hypothesis is supported by the different attitude of the lay public and the Internet toward stevia, a sweetener extracted from a plant that grows in South-Central America and that was recently approved by the EFSA, although it has been used in Japan for approximately 30 years and in the USA since 2008.

In fact, stevia is featured with positive attributes in all of the first 50 websites that appear when typing its name in Google (there are approximately 15 million sites that mention stevia, i.e., ten times those that feature aspartame).

The notion that "nature is our friend" and that, therefore, natural substances are devoid of deleterious effects, appears to be shared by many, at least on the Internet. This is at times odd, especially considering that some plants and their derivatives are notoriously toxic.

It is necessary to counterbalance this approach, which is not very scientific, to the issue of LCS and their safety. One example is the meeting "No-Calorie Intense Sweeteners: Focus on Safety of Use", organized by the Nutrition Foundation of Italy and supported by the Italian Ministry of Health, in which several experts convened to evaluate the available evidence. One such expert is Dr. J.C. Larsen, who has also written a timely and updated article on this topic for the current issue of NutraFoods.

Clearly, it is difficult to reverse the current trend, which has seen the scientific community and national and international regulatory bodies lose part of their authority and reliability. If we want to pursue such a goal, we will need to work hard and patiently, and will only see results in the mid-term.

One starting point should be to contain the scientific debate within the realm of scientists, who speak the same language. This would avoid confusion in the media and the generation of opinions not based on scientific evidence but rather "ideological" that, once established, are very difficult to change.

Andrea Poli Editor in Chief 